\section{Lasting Biological Effects of Early Influences}

\section{RENÉ DUBOS}

\author{
The Rockefeller University, New York, New York 10021
}

In his essay "Uses of Great Men" Ralph Waldo Emerson wrote a statement that has a direct bearing on the topic I wish to discuss in the present paper: "There are vices and follies incident to whole populations and ages. Men resemble their contemporaries even more than their progenitors."

As a moralist, Emerson was primarily concerned with the intellectual and moral attributes of human beings; his aphorism is just as valid for anatomic and physiological attributes. We resemble our progenitors because we derive from them our genetic endowment; but genes do not really determine the traits by which we know a person. They only govern the responses that the person makes to environmental stimuli. Individuality progressively emerges from these responses.

Whereas the genetic pool of a population remains essentially constant, the environment changes rapidly. We resemble our contemporaries, because the phenotypic expression of the genetic endowment is determined by the environmental forces that impinge more or less simultaneously on all the members of a given generation in a given social milieu.

The phenotype is constantly being molded by the environment throughout the whole life span. But early influences certainly play the most important role in converting genetic potentialities into phenotypic reality. As commonly used, the phrase "early influences" denotes the conditioning of behavior by the experiences of early life.
Early experiences, however, do more than condition behavioral patterns; they also affect, profoundly and lastingly, other biological characteristics such as initial growth rate, efficiency in the utilization of food, anatomic structures, physiologic attributes, maximum adult size, resistance to infection, response to various forms of stimuli -in brief, almost every phenotypic expression of the adult.

A few examples from contemporary life will suffice to illustrate the effects of early influences on human populations.

Japanese teenagers are now much taller than their parents and differ in behavior from prewar teenagers, not as a result of genetic changes, but because the post-war environment in Japan is very different from what it was in the past. A similar phenomenon is observed in the settlements of Israeli kibbutz. The kibbutz children are given a diet and sanitary conditions as nearly optimum as can be devised. Early in their teens, as a result, they tower over their parents, who originated from crowded and unsanitary ghettos in Central and Eastern Europe.

The acceleration of growth in Japan and in the Israeli kibbutz constitutes but a particular case of a constant trend toward earlier maturation of children in Westernized countries. This is evidenced by greater weights and heights of children at each year of life and by the earlier age of the first menstrual period. In Norway, for example, the mean age of menarche has fallen from 17 years in 1850 to
13 in 1960; similar findings have been reported from Sweden, Great Britain, the United States, and other affluent countries.

Growth is not only being accelerated; the final adult heights and weights are greater as well as being attained earlier. Some 50 years ago, maximum stature was not being reached, in general, until the age of 29; commonly now it is reached about 19 in boys and 17 in girls. With regard to the age of puberty, the change seems to consist in the restoration of the developmental timing that had prevailed in the past and that had been greatly retarded by the ways of life at the beginning of the 19th century.

The factors responsible for these dramatic changes in the rate of anatomic and sexual maturation are not completely understood. There are good reasons to believe, however, that improvements in nutrition and control of childhood infections have played a large part in the acceleration of development, and that this change in turn has been responsible for the larger size achieved by adults.

I shall briefly describe experimental models that illustrate the effects of early influences on the development of laboratory animals.

Pioneering investigations on the lasting biological effects of early postnatal influences were carried out in England during the 1960's by $R$. A. McCance and Elsie M. Widdowson of Cambridge University. These investigators compared the growth of rats suckled in small litters (three young per lactating 


\section{R. DUBOS}

female) with that of comparable animals suckled in large litters (18 young per female). They found that the rats of the latter group became much smaller adults than those of the former group, even though the animals of both groups were given unlimited food after weaning. It can be assumed that the animals raised in small litters developed more rapidly than the others because they enjoyed a nutritional advantage during the lactation period. As McCance and Widdowson pointed out, however, the interpretation of their findings may be more complex than appears at first sight. The design of their experiments did not rule out the possibility that infectious and psychological disturbances occurred when lactating females had to feed unusually large numbers of young. Such disturbances might have played a role in the retardation of growth observed in this group.

In our own studies now to be described, an attempt was made to eliminate or minimize the disturbing effect of indirect, non-nutritional factors. To this end, the experiments were conducted with specific-pathogen-free (SPF) mice so as to avoid the activation of latent pathogens by nutritional deficiencies. In all cases, the litter size was reduced to eight young, which is physiologically normal for mice. Whenever possible the animals were put on the experimental diets two weeks before mating so as to eliminate the behavioral disturbances that commonly occur at the time of a dietary change.

The principle of the experiments consisted in introducing the conditioning factor (infection, nutritional deficiency, or behavioral disturbance) very early in the life of the animal-either during gestation or lactation, or both. Immediately after weaning, all young animals were placed under exactly the same optimum conditions of husbandry and kept undisturbed during their whole life span. This made it pos- sible to recognize the lasting effects of the early manipulation on characteristics such as rate of growth, maximum adult size, longevity and resistance to various forms of stress.

SPF mice weigh more at weaning time than mice of the same genetic origin raised under less favorable conditions. Furthermore, they reach larger adult size and exhibit more desirable physiological characteristics as well as greater resistance to various forms of stress. It might be assumed that these differences are the result of unrecognized genetic changes that occurred during the development of the SPF colony. However, this explanation has been ruled out by several types of experiments.

In one type of experiment, newborn SPF mice were contaminated per os one or two days after birth with fecal material obtained from ordinary mice that had been raised on a commercial farm and were "healthy" according to usual criteria. The contamination could be so controlled that it did not cause any obvious sign of disease, not even diarrhea. Yet the contaminated animals were smaller than the uncontaminated controls at weaning time. Even more interestingly, they grew into smaller adults. Depression of weight was at times so profound that the contaminated animals were almost runts at the time of weaning.

The following facts strongly suggest that this growth-depressing effect is associated with viral multiplication in the intestinal tract. When SPF mice are contaminated per os shortly after birth with certain bacterial cultures isolated from the intestinal contents of adult ordinary mice, these bacteria multiply extensively throughout the gastrointestinal tract and persist at extremely high levels until weaning time. However, such bacterial infections do not affect significantly either weaning weight, growth rate, or maximum adult weight.

In contrast, weight depression could be consistently brought about by contaminating newborn SPF mice per os with bacteria-free filtrates of homogenates of intestines from ordinary mice. The weight-

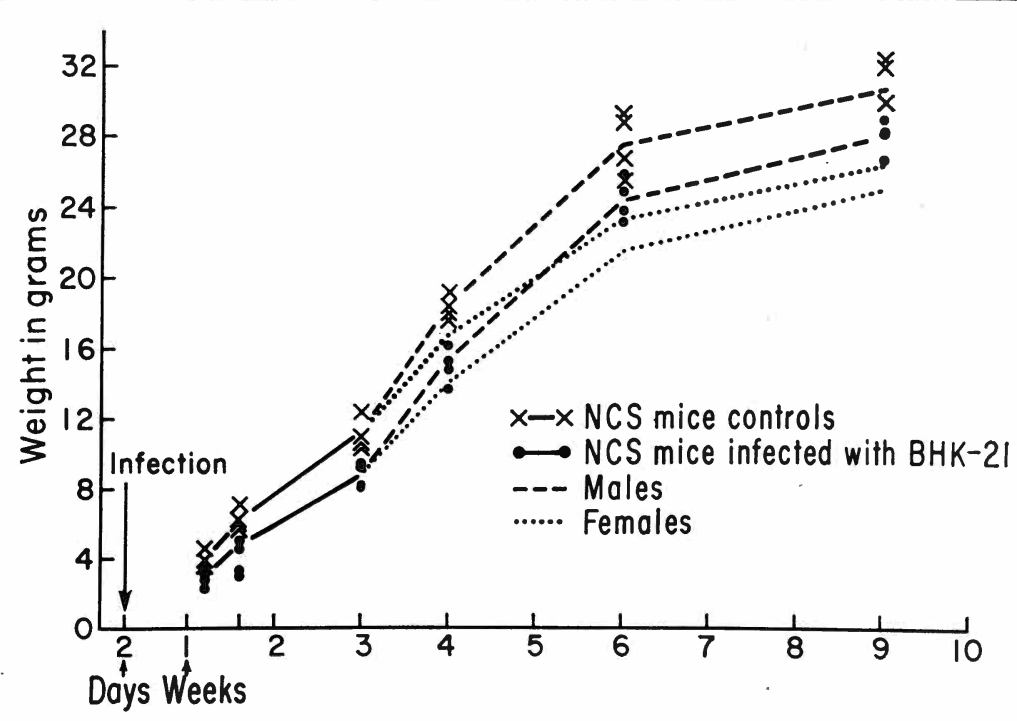

Fig. 1-Weight curves of SPF mice contaminated orally on second day of life with fifth passage of BHK-21 tissue culture infected with enteric virus.

Each point represents the average for each box of animals; each curve corresponds to averages for approximately 15-20 animals. 
depressing agent passed through Millipore discs of $0.45 \mu$ and $0.22 \mu$ porosity but was held back at $0.10 \mu$ porosity.

On several occasions, but not consistently, bacteria-free filtrates capable of depressing the weight curve of SPF mice produced alterations in the appearance of tissue cultures of baby hamster kidney cells (BHK-21) and mouse embryo cells. When tissue cultures so infected were introduced into newborn SPF mice, the weight of these animals was depressed early and lastingly (Fig. 1 and 2).

Only very young SPF mice (preferably less than three days old) proved susceptible to the weightdepressing effect of the filtrates of intestine homogenates or of infected tissue cultures prepared therefrom. After oral contamination, it took approximately one week before the intestinal homogenate obtained from contaminated animals exhibited a high level of weight-depressing activity.

The growth-depressing effect could be transmitted from one generation to the next by mating SPF mice that had been contaminated shortly after birth and were consequently smaller than control SPF animals (Fig. 3).

A lasting effect on growth can be produced also in the absence of contamination by feeding the mouse dam a restricted or deficient diet, either during gestation or, after the birth of her young, during the lactation period.

Lasting depression of growth has been achieved, for example, by lowering the content of the dam's diet in magnesium, or in lysine and threonine. The growth-depressing effect so achieved persisted throughout the whole life span of the young, even though the latter were given, at weaning time and constantly thereafter, unlimited amounts of an optimum diet. These findings are relevant to human situations, since the diets of most underprivileged people consist chiefly of plant products,

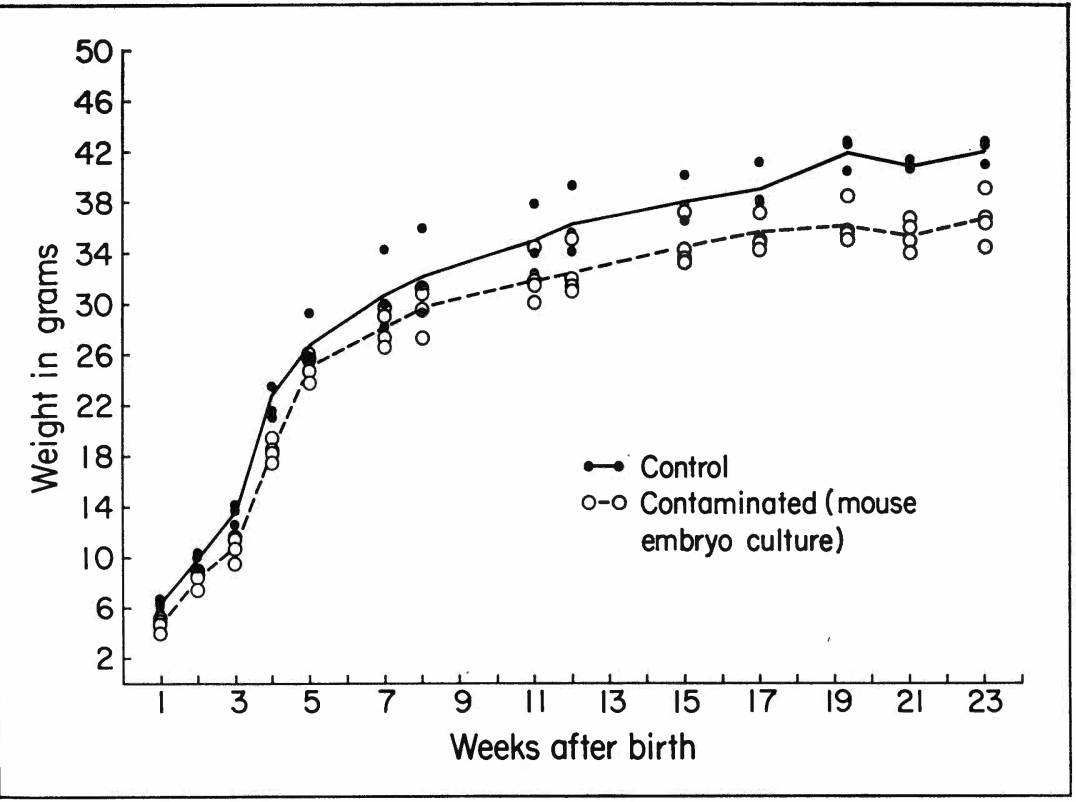

Fig. 2-Weight curves of SPF mice contaminated two days after birth with mouse embryo infected cells.

Each point represents the average weight for each box of animals; each curve corresponds to approximately 15-20 males.

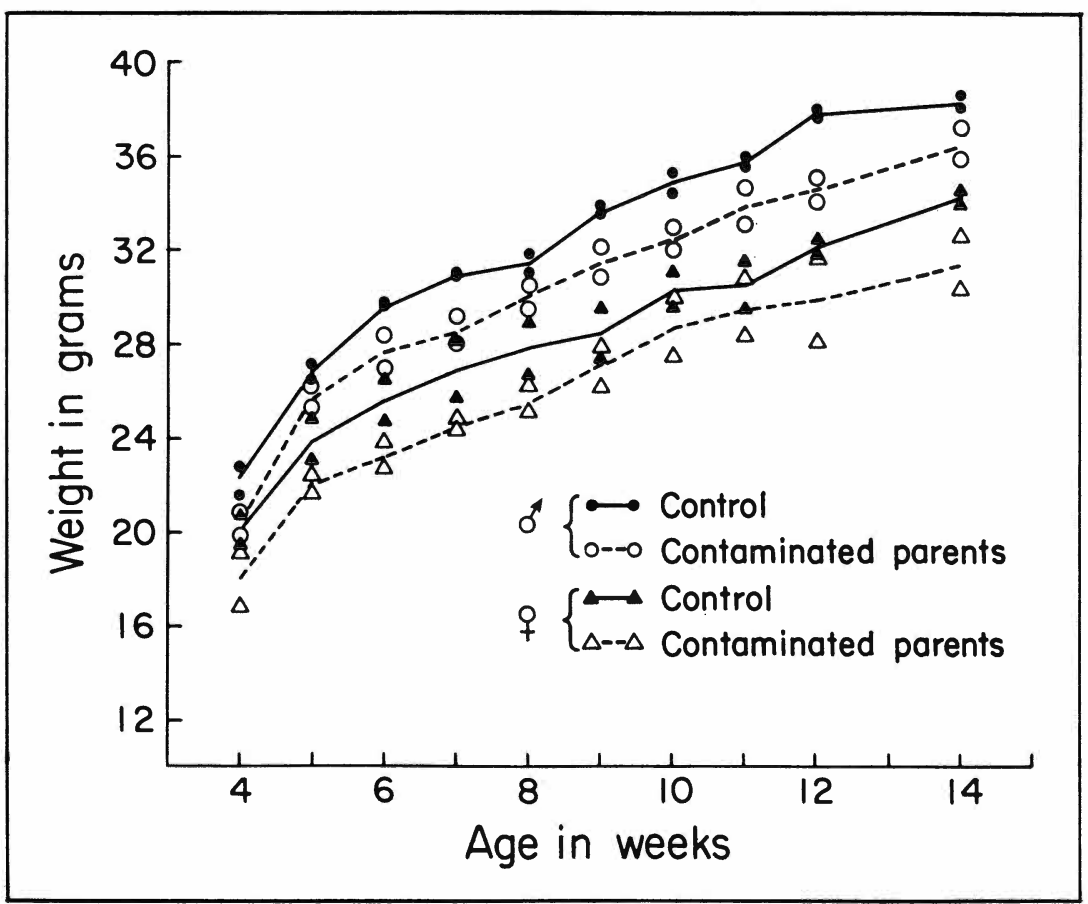

Fig. 3-Weight curves of SPF mice born from uncontaminated and contaminated parents.

Each point represents the average weight for each box of animals; each curve corresponds to approximately 20 animals. 
which are commonly deficient in certain amino acids and minerals (Fig. 4 and 5).

Depression of growth resulting from lysine and threonine deficiency during gestation or lactation did not seem to affect adversely the health of the young, or to decrease their longevity. In fact, the results of two experiments in which the animals nursed by mothers on different diets were kept undisturbed and on optimum diets throughout their whole life span suggest that the smaller animals had a greater average life expectancy than the larger ones (Fig. 6).

Even in the absence of infection and of malnutrition, SPF mice naturally exhibit individual differences in weaning weight, growth rates, and adult size. We have observed, on the other hand, that the young of each particular mother are remarkably uniform in size. It was assumed that the differences from litter to litter, and the uniformity within each litter, were determined by the genetic endowment of the young. But this explanation does not account for the facts, as shown by the unexpected results of the following experiments.

Newborn SPF mice of exactly the same age were pooled one day after birth. They were then randomly reallocated to the various mothers, each foster mother receiving eight young born the same day. The foster mothers were all fed an optimum mixed natural diet and placed under favorable laboratory conditions. They readily accepted the young allocated to them, and these began to nurse immediately. Within a very few days, however, marked differences could be detected in the growth of the newborn mice. Contrary to expectation, the differences were small within each group nursed by a given foster mother, while they were much more pronounced from one group to the other (Fig. 7).

The pattern of differences was very obvious after two weeks and particularly at the time of weaning.
Furthermore, the relative rank of weights remained the same after weaning, even though all mice received from then on the same optimum diet in the same room. Figure 7 illustrates the range of differences within litters and between litters at different periods after separation of the young from their foster mothers. Adult size, as well as weight during lactation and at weaning time, obviously reflected the experience of the very first days of life.

The design of this type of experiment rules out the possibility that the differences in weights and growth rates from litter to litter could be caused by differences in genetic constitution among the young animals, since these had been pooled and randomized; neither could the differences in growth be traced to prenatal influences, since the animals had been randomized after birth. Uniformity within a litter and differences from litter to litter were due, therefore, to the influence of the foster mother during lactation. As far as can be judged, the quantity and quality of milk did not differ from mouse to mouse, but the foster mothers differed markedly in their "mothering" behavior (attention to the young, nest building, etc.). What is certain, in any case, is that the early experience derived from the foster mother conditioned the young so profoundly that the effects persisted long after the end of lactation into adult age.*

No systematic study has yet been made of the mechanisms through which early influences exert such profound and lasting effects. But a

* The weight depression produced by the virus mentioned on pages 53 and 54 is still evident 18 months after oral contamination of the newborn animals. Furthermore, new experiments with the same virus preparation have resulted in a weight depression much more profound than that recorded in Figures 1, 2, and 3. The wall of the small intestine is very much thickened in these infected ani-

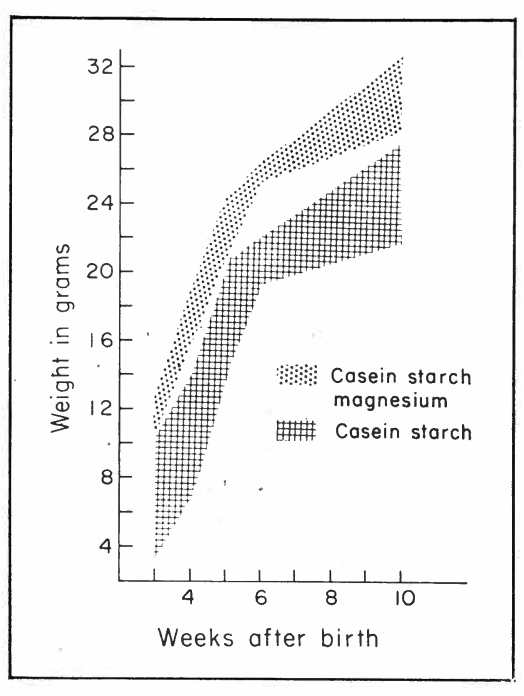

Fig. 4-SPF mice fed pellets until three days before delivery. From then on, and throughout lactation, half of them were fed casein-starch diet; the other half the same diet supplemented with magnesium chloride. All animals transferred to pellets after weaning.

The graphs show the weight range for each group (48 animals per group) until the fourth week of life, then for the males only.

few facts point to useful working hypotheses.

Adult animals which have suffered from viral enteric infections or nutritional deprivation early in life exhibit a markedly decreased ability to absorb and utilize the food which is given them later; the incorporation of amino acids is particularly depressed. Such anabolic defects persist long after the early experience that caused them initially.

There is evidence also that certain viral infections, nutritional deficiencies and behavioral disturbances interfere with the synthesis

mals, which continue to exhibit malabsorption and deficiency in amino acid assimilation long after oral contamination.

Similar phenomena have been observed in germ-free chicks (Eyssen and De Somer, 1967). Also relevant to this study is a paper by Berg, Simms, and Everitt (1963), which demonstrates an effect of early nutrition on longevity. 
of various hormones (growth hormone, for example) at critical periods of development.

Other preliminary studies have revealed that the numbers of certain types of cells are determined early in life and are conditioned by various influences. For example, mice and rats nursed by mothers fed a restricted or deficient diet appear to have unusually small numbers of lipogenic cells. This may explain why they do not become obese in old age, as is the tendency among animals produced and nursed by dams fed a rich diet, and also why they seem to have a long life span.

Early influences also affect the development of neural systems and of behavioral patterns, as can be

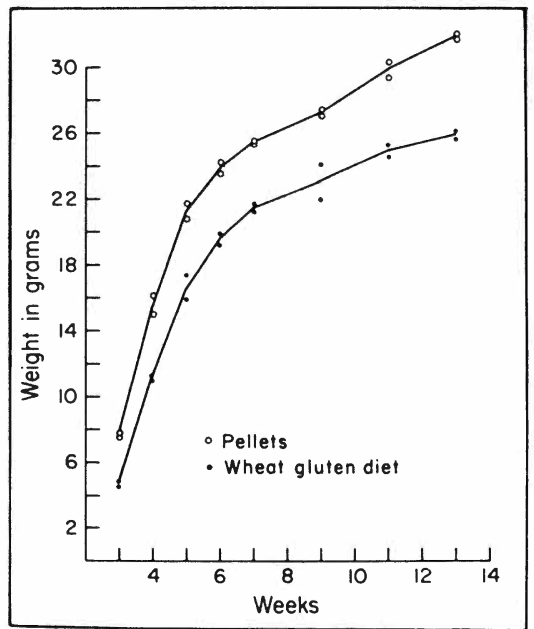

Fig. 5-SPF mice were fed pellets and mated. Approximately one week before delivery of the young, half of the pregnant animals were changed to wheat gluten diet and maintained on this diet until their young were weaned at three weeks of age. All the animals were then fed pellets for the rest of their lives.

The two graphs in this figure include males and females of the two progenies (two cages of 16 animals each per group). Males and females were separated after the fifth week of life, but the weights of all the animals from each cage were pooled and averaged until the 14th week of life. readily demonstrated in many species of animals. The classical "imprinting" of birds is almost a caricature of this phenomenon; most techniques of animal training make practical use of the possibility to control behavioral organization during early life. The molding of the neural apparatus and associated conditioning of behavior are of particular importance in man, because the nervous system of the human brain is incompletely developed at birth. Mentally, the child develops as he responds to environmental stimuli.
In man, as in animals, there are critical periods of mental as well as physical development, but the timing and duration of these critical periods have not been defined. It is certain, in any case, that the manifestations of mental development are conditioned by the metabolic factors that govern anatomic and physiologic growth and by the nature of the stimuli to which the child is exposed. The social environment during early life, therefore, affects profoundly, and in most cases irreversibly, the fate of the person.

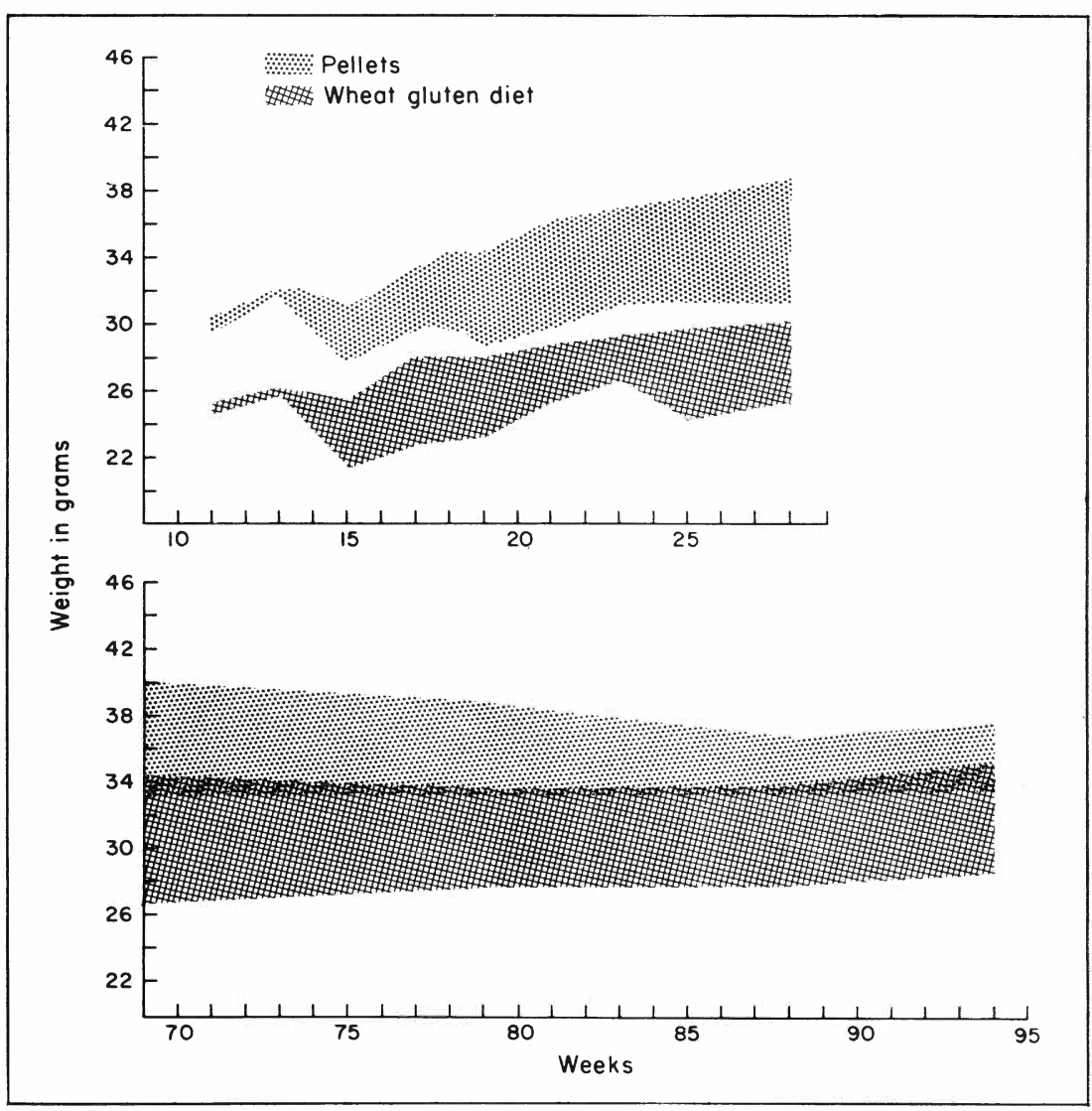

Fig. 6-This experiment is the same as that illustrated in the text-Figure 5; from the 14th week of life, the males were transferred to individual cages; the females were discarded.

The loss of weight in the 15th week was caused by the fact that the animals had been transferred to individual cages on wire grids. The fluctuations of the two curves thereafter express the effects of unidentified environmental factors.

The graphs indicate the range of weight variation for each of the two diet groups.

Deaths began to occur in the pellet groups after two years, the heaviest animals dying first. None of the animals survived beyond two years and seven months. 
Experiments with animals have proven that an environment rich and diversified in stimuli affects neural and behavioral development, as measured by exploratory activity, learning ability, and even by chemical and enzymatic properties of the brain.

An impoverished environment results in behavioral deficiency. The same principles certainly apply to human beings.

Human potentialities, whether physical or mental, can be actualized only to the extent that circumstances favor their phenotypic expression. In consequence, diversity within a given society is an essential component of true functionalism. The latent potentialities of human beings have a better chance to emerge when the social environment is sufficiently diversified to provide a variety of stimulating experiences, especially for the young.

As more and more persons find it possible to express their biological endowments under a variety of conditions, society becomes richer, and civilizations continue to unfold. In contrast, if the surroundings and ways of life are highly stereotyped, the only components of man's nature that flourish are those adapted to the narrow range

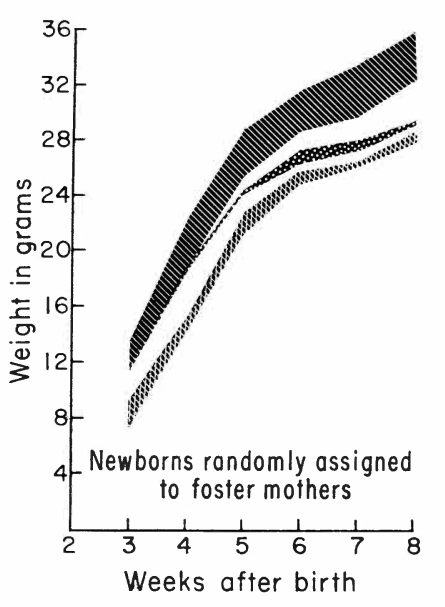

Fig. 7-SPF mice pooled on second day of life, then immediately allocated randomly to foster mothers (eight per mother). All animals fed pellets. of prevailing conditions. Hence, the dangers of many modern housing developments-designed as if their only function was to provide disposable cubicles for dispensable people.

Irrespective of their genetic constitution, most young people raised in a featureless environment, and limited to a narrow range of life experiences, will be crippled intellectually and mentally. For this reason, we must shun uniformity of surroundings as much as absolute conformity in behavior. Creating diversified environments may result in some loss of efficiency; but diversity is vastly more important than efficiency, because it is essential to the germination of the seeds now dormant in man's nature.

In conclusion: The environment does more than affect our well-being in the here and now. It molds the young, physically and mentally, and thereby determines almost irreversibly their future and the evolution of society.

\section{References}

Berg, B. N., H. S. Simms ANd A. V. EVERITT. Nutrition and longevity in the rat. V. Weaning weight, adult size, and onset of disease. J. Nutr. 80:255-262, 1963.

Dubos, R., R. W. Schaedler AND R. Costello. Lasting biological effects of early environmental influences. I. Conditioning of adult size by prenatal and postnatal nutrition. J. Exp. Med. 127:783-799, 1968.

EYSSEN, H. AND P. DE SOMER. Effects of Streptococcus faecalis and a filterable agent on growth and nutrient absorption in gnotobiotic chicks. Poultry Sci. 46:323-332, 1967.

Newton, G. AND S. Levine (eds.) Early Experience and Behavior. Springfield, Ill.: C. C. Thomas, 1968.

Seravalli, E. AND R. Dubos. Lasting biological effects of early environmental influences. II. Lasting depression of weight caused by neonatal contamination. J. Exp. Med. 127:801-818, 1968. 